\title{
Evacuating Uterine Contents before Operative Hysteroscopy in Patients With Active Uterine Bleeding: A Randomized Clinical Trial
}

\author{
Manizheh Sayyah-Melli ${ }^{* \oplus}$, Maryam Kazemi-Shishavan ${ }^{1,2}$, Nooshin Behravan', Parvin Mostafa \\ Gharabaghi', Vahideh Rahmani'
}

\begin{abstract}
Objectives: Concurrent bleeding or existing clots usually obscure the vision field and decrease the hysteroscopy success rate. Therefore, any efforts made to have a clear view during the hysteroscopy will improve the diagnostic or treatment outcomes. We examined the effect of preoperative clot evacuation on hysteroscopy related outcomes.

Materials and Methods: In this parallel-group randomized clinical trial conducted in Al-Zahra hospital, Tabriz, Iran, 114 women with uterine bleeding were randomly assigned to receive either clot evacuation before standard operative hysteroscopy or after that from December 2018 to September 2019. The study outcomes were the clarity of vision, amount of bleeding, the volume of required distension media, duration of the procedure, and postoperative complications.

Results: There were statistically significant differences in the frequency of the clear vision $(P<0.001)$, the severity of bleeding, mean procedure time $(P<0.001)$, mean used distension media and the mean postoperative hematocrit levels in favour of women with pre-hysteroscopy intrauterine evacuation. There was no difference in in-hospital stay and anaesthetic complications among the two groups. The procedure was successfully performed on all participants of both groups with no post-operative complications.

Conclusions: Removal of clots and other uterine contents before the insertion of the hysteroscope rendered better and faster access to the uterine wall to observe existing abnormalities. This additional surgical step could significantly impact surgical and clinical outcomes.

Keywords: Hysteroscopy, Uterine bleeding, Metrorrhagia, Endoscopy
\end{abstract}

\section{Introduction}

Abnormal uterine bleeding significantly impacts women of childbearing age $(1,2)$ and postmenopausal women (3). Depending on the underlying etiology, the surgeon may decide on a less invasive surgical procedure or not a surgical treatment. Diagnostic, operative hysteroscopy is the practice of choice to evaluate intrauterine pathologies in patients with premenopausal $(4,5)$ and postmenopausal bleeding (6). Direct visualization of the entire uterine cavity provides an opportunity to perform a biopsy of suspicious lesions or surgical resection (7). However, operative hysteroscopy may be associated with complications such as fluid overload, uterine perforation, and bleeding (8). Several strategies facilitated the procedure and improved prognosis (9-11). There is evidence of using gonadotropin agonists $(12,13)$ and letrozole (14) as a preoperative intervention. However, the quality of the evidence in some of these studies was low. Other interventions, such as the administration of epsilon aminocaproic acid (15), oxytocin (16), or an intrauterine balloon, are used to reduce the amount of bleeding and the rate of complications during and after the hysteroscopic procedures (17). The suction curettage efficiency during operative hysteroscopy has facilitated the removal of relatively large, multiple, or single endometrial polyps and submucous uterine myomas (18). Direct visualization by a hysteroscope could facilitate a more efficient vacuum aspiration of the retained conception tissue and reduce surgical complications (19). In a randomized controlled trial study, carbon dioxide was used as a distension media in patients without excessive bleeding at the time of at hysteroscopy (20). Furthermore, a balloon catheter has been used successfully for cervical ripening in young women before operative hysteroscopy (21).

There is no evidence regarding optimizing visual quality during hysteroscopic procedures in non-pregnant women with uterine bleeding at the starting of the procedure. Therefore, this study was designed to evaluate the effects of intrauterine contents evacuation before starting the operative hysteroscopy on efficient hysteroscopic procedures. 
Key Messages

- A clear view during the hysteroscopy improves the diagnostic or treatment outcomes. This study showed that removing clots and other uterine contents before the insertion of the hysteroscope rendered better and faster access to the uterine wall and improved the clarity of vision, amount of bleeding, and distension media volume and duration of the procedure.

\section{Materials and Methods}

Study Design and Participants

We performed a parallel-group randomized clinical trial involving women with abnormal uterine bleeding recruited from December 2018 to September 2019 at the Oncology Center of Alzahra Teaching hospital, Tabriz, Iran. Women with active uterine bleeding due to uterine pathology or misoprostol use were eligible to participate in the study. Women who had coagulopathy disorders, known cervical or uterine malignancy, or marked cervical stenosis, genital tract infections, received treatment affecting endometrium growth have been excluded. Withdrawal criteria were surgical or anesthetic complications during the hysteroscopy procedure.

\section{Interventions}

All women had undergone Pap smear, transabdominal and transvaginal ultrasonography, hematological assessments, and endometrial pipelle sampling before the operation. Each participant's information was recorded in the data collection forms before the procedure. Participants were randomly divided into the case and control groups using numbered envelopes using RandList version 2.1 (Datinf $\mathrm{GmbH}$, Tübingen, Germany). The lead researcher used this sequence to determine the method for participants immediately after the general anaesthesia. All women underwent general anaesthesia according to the same protocol, and the same surgeon performed all procedures. In the case group, the surgeon dilated the uterine cervix with the Hegar dilator and removed clots and contents of the uterine cavity with ring forceps. Then, the resectoscope was inserted to examine the entire uterine cavity and remove visible lesions. All procedures were performed with glycine $1.5 \%$ as a distension medium, monopolar instrumentation, and during the follicular phase in the fertile women. In the control group, the surgeon started with resectoscope insertion regardless of uterine bleeding, and later procedures were the same as the case group.

\section{Outcomes and Data Collection}

The amount of bleeding during surgery was recorded using a Likert scale [zero (no bleeding) to 5 (severe bleeding)] as agreed by both the surgeon and the circulating nurse. The primary outcomes were the clarity of the view during the procedure, duration of surgery, amount of bleeding and the secondary outcomes included hematocrit levels 6 hours after surgery and primary complications.

\section{Sample Size}

Due to the lack of similar studies, Cohen's estimate of medium effect size (0.3) was considered desirable for calculating the sample size required to reject the null hypothesis of 0.05 with a power of 0.8 using the $G^{*}$ Power 3.0.10 software. We computed the required sample size using the 2 degrees of freedom chi-square test that at least 54 people were needed in each group. We estimated 114 for the final recruitment sample size considering a $10 \%$ probability of not participating or losing follow-up.

\section{Blinding}

The statistician was blinded to the grouping until the final report.

\section{Statistical Analysis}

Intention to treat analysis was performed, and independent samples $t$ test, paired $t$ test, and Mann Whitney $\mathrm{U}$ test were used for data analysis. Pearson Chi-square was used for the comparison of categorical variables. Data analysis was performed using IBM SPSS software (IBM Corp, Armonk, NY, USA) version 25 at the significance level of 0.05 .

\section{Results}

We approached 125 eligible participants, six declined to participate, and five did not meet the study's inclusion criteria. One hundred and fourteen were randomly allocated to case and control groups. None of the participants was lost to follow-up; therefore, all the participants were included in the analysis (Figure 1). In addition, the participants' baseline characteristics and their indications for hysteroscopy were presented in Table 1.

We used a $\chi^{2}$ test with adjusted standardized residuals and corresponding adjusted significance levels to compare bleeding categories in the case and control groups. A smaller percentage of the participants in the case group experienced "Severe bleeding" $(P<0.0001)$ and "Moderate bleeding" $(P<0.001)$. Moreover, the proportion of "None bleeding" occurred more in the case group participants $(P<0.001)$. There was no statistically significant difference between the two groups in the "Mild bleeding" category $(P=0.635$; Figure 2).

There was a significant association between the case group and the vision quality categories $\left[\chi^{2}(2)=50.61\right.$, $(P<0.001)(\phi=0.66, P<0.001)]$. The "clear vision" depicted in figure 3 was observed more in the case group (91.2\%) than the control group $(26.3 \%)(P<0.001)$. Compared to the controls (64.9\%), "blurred view" was significantly less frequent in the case group $(5.3 \%)(P<0.001)$. The rate of "no clear view" was not statistically different between the two groups ( $P=0.241$; Figure 3$)$.

The comparison of procedure duration, media volume 


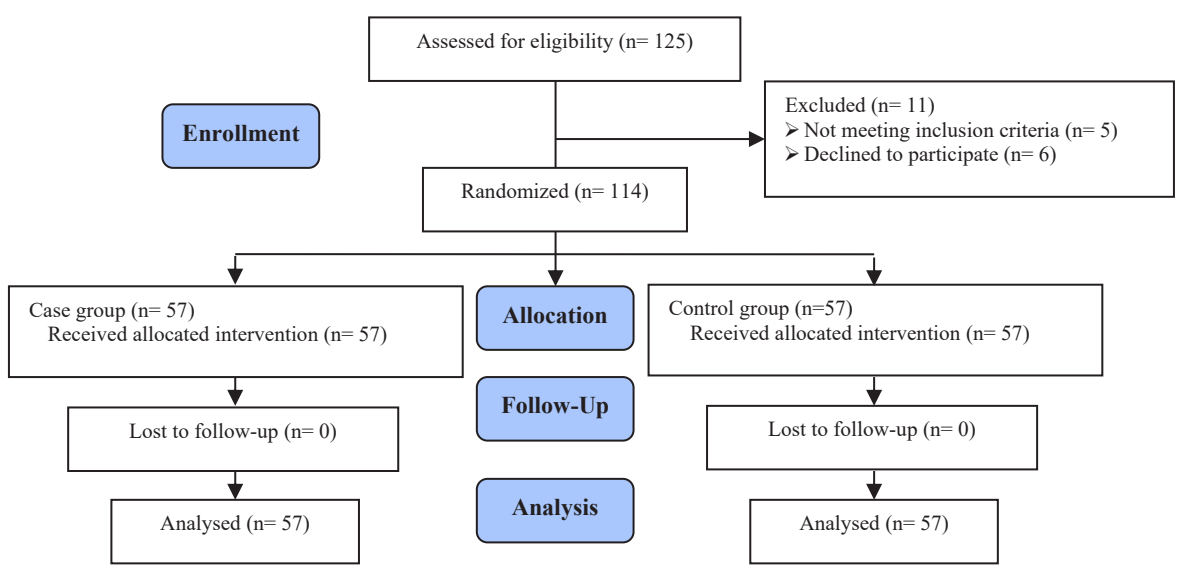

Figure 1. Consort Flow Diagram of the Study.

use, and admission duration between the two groups is summarized in Table 2. The procedure continuation was considerably shorter in the case group $(U=765.5$, $(P<0.001)$. In addition, the Glycine volume used in the case group was significantly lesser than the amount used in the control group ( $\mathrm{U}=754.0, P<0.001)$.

The postoperative hematocrit reduction was higher in the control group $[95 \%$ CI $(-3.00,-1.88)]$ (Table 2). There were no complications associated with general anesthesia or surgery in either of the groups. However, 4 (7.0\%) of participants in the case group and $1(1.8 \%)$ in the control group reported nausea after recovery $(P=0.182)$.

\section{Discussion}

In this randomized trial, our study findings showed that removing the clots and uterine contents beforehysteroscopy

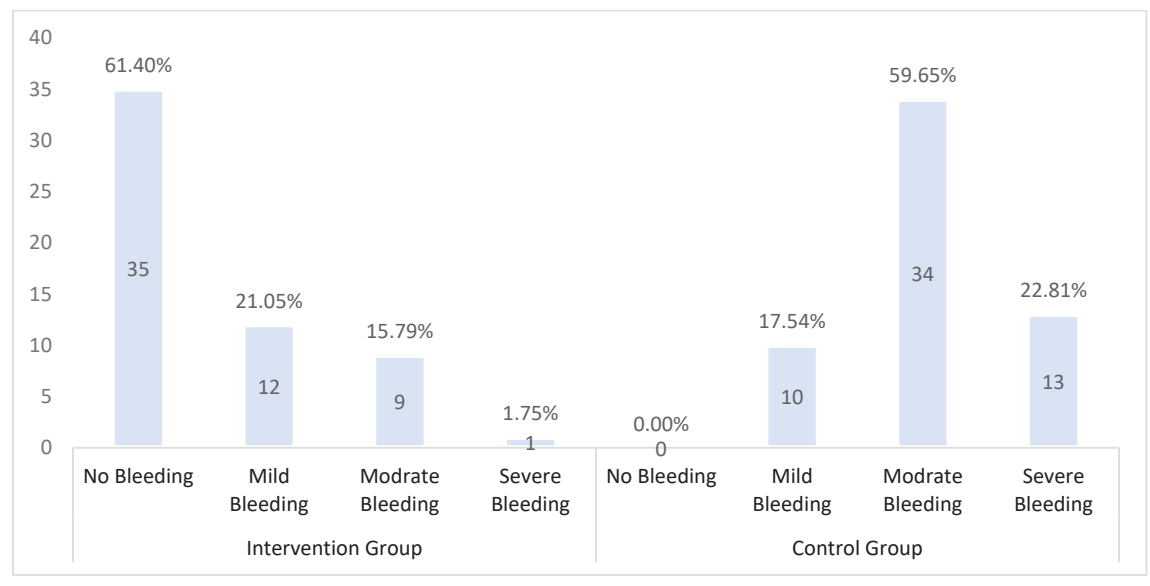

Figure 2. Frequency of Bleeding Categories During the Hysteroscopy in the Study Groups.

Table 1. Demographic Characteristics of the Study Participants

\begin{tabular}{|c|c|c|c|c|c|}
\hline Variables & Total Sample $(\mathrm{N}=114)$ & Case Group $(n=57)$ & Control Group $(n=57)$ & $\operatorname{tor} Z$ or $\chi^{2}$ & $P$ Value \\
\hline Age (y), Median (IQR) & $44(39-50)$ & $44(37-51)$ & $46(40-50)$ & -0.434 & $0.664^{\mathrm{a}}$ \\
\hline Married (y), Mean (SD) & $23.30(10.12)$ & $23.33(11.46)$ & $23.28(8.6)$ & 0.025 & $0.980^{\mathrm{b}}$ \\
\hline Gravidity, Median (IQR) & $2.00(2.00-3.50)$ & $2.00(2.00-3.00)$ & $3.00(2.00-4.00)$ & -1.40 & $0.159^{\mathrm{a}}$ \\
\hline Parity, Median (IQR) & $2.00(2.00-3.00)$ & $2.00(2.00-3.00)$ & $2.00(2.00-3.00)$ & -1.04 & $0.295^{\mathrm{a}}$ \\
\hline \multicolumn{6}{|c|}{ Hysteroscopy indication, No. (\%) } \\
\hline Leiomyoma & $77(67.5)$ & $35(61.4)$ & $42(73.7)$ & & \\
\hline Endometrial polyp & $29(25.4 .3)$ & $19(33.3)$ & $10(17.5)$ & 3.92 & $0.140^{c}$ \\
\hline Endometrial thickening & $8(7.0)$ & $3(5.3)$ & $5(8.8)$ & & \\
\hline
\end{tabular}

${ }^{a}$ Mann-Whitney $U$ test, ${ }^{b}$ Independent samples $t$-test, ${ }^{c}$ Chi-square test. 


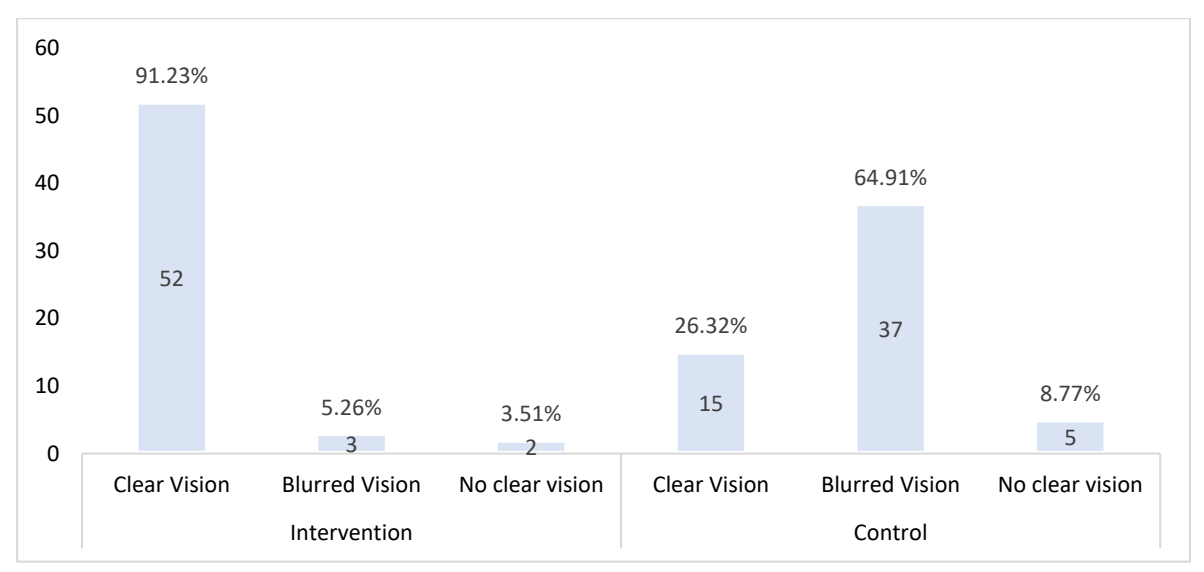

Figure 3. Frequency of Hysteroscopy Vision Categories in Case and Control Groups.

facilitates the procedure efficiency by providing a more transparent view. In addition, this technique resulted in a shorter duration of surgery $(P<0.001)$, a considerable reduction in used distension media $(P<0.001)$ and less blood loss $(P<0.001)$. Hysteroscopy is a standard method for evaluating abnormal uterine bleeding and is a vastly used procedure for removing intrauterine polyps, submucosal myoma, pregnancy remnants, and cesareaninduced pathologies (22-24).

Clear vision during hysteroscopic procedures is crucial for favourable outcomes. In addition, the theory that blind removing of polyps may result in malignant cells at the base of the tumor (25) does not apply in this method because the hysteroscope then enters the uterine cavity to remove the remaining mass.

Several researchers used notable drugs to reduce complications of hysteroscopy, such as oxytocin, misoprostol, vasopressin, bupivacaine and epinephrine, mesna, and intrauterine injection aminocaproic acid. In addition, some studies used pericervical tourniquet, cervical balloon catheter, and Foley balloon tamponade in this matter. However, these cases' effectiveness has not been fully clarified $(16,17,21)$.

Researchers have also intended to modify standard hysteroscopic procedures for better outcomes. For example, Murakami et al safely used vaporization and prostaglandin F-2 alpha to reduce the operation time and hysteroscopic complications in women with submucosal myoma (26). In another study, curettage suctioning use during myomectomy has reduced the complication rates (18). Moreover, the successful use of hysteroscopy to evacuate the remnants of pregnancy is reported in various studies. Zhu et al used forceps to remove remnants of the placenta and clots before hysteroscopy to reduce thermal and electrical damage during the procedure (27).

Our results indicate that removing intrauterine contents with concurrent active uterine bleeding is reasonable to view the inner uterine wall. Based on the findings for shorter procedure duration and reduction in Glycine volume use, this procedure, resulting in an efficient and practical method. A clear hysteroscopic view increases the probability of locating lesions; thus, the success rates

Table 2. Duration of Surgery, Distending Media, Duration of Hospital Stay, and Hematocrit Levels in Both Study Groups

\begin{tabular}{|c|c|c|c|c|c|}
\hline \multirow{2}{*}{ Variables } & \multirow{2}{*}{ Groups } & \multirow{2}{*}{ Median } & \multicolumn{2}{|c|}{ IQR } & \multirow{2}{*}{$P$ Value* } \\
\hline & & & Q1 & Q3 & \\
\hline \multirow{2}{*}{ Duration of Surgery (min) } & Case & 6.00 & 4.75 & 24.00 & \multirow{2}{*}{$<0.001$} \\
\hline & Control & 10.00 & 6.00 & 15.00 & \\
\hline \multirow{2}{*}{ Distending Media (mL) } & Case & 1000.00 & 725.00 & 2000.00 & \multirow{2}{*}{$<0.001$} \\
\hline & Control & 2500.00 & 1750.00 & 3750.00 & \\
\hline \multirow{3}{*}{ Hospitalization(days) } & Case & 1.00 & 1.00 & 1.00 & \multirow{2}{*}{0.081} \\
\hline & Control & 1.00 & 1.00 & 1.00 & \\
\hline & & Mean \pm SD & Mean Di & ifference) & \\
\hline \multirow{2}{*}{ Hct. (Before Procedure) (\%) } & Case & $37.46 \pm 3.62$ & \multirow{2}{*}{\multicolumn{2}{|c|}{$0.58(-0.67-1.83)$}} & \multirow{2}{*}{0.360} \\
\hline & Control & $36.88 \pm 3.10$ & & & \\
\hline \multirow{2}{*}{ Hct. (After Procedure) (\%) } & Case & $36.26 \pm 3.68$ & \multirow{2}{*}{\multicolumn{2}{|c|}{$3.32(2.09-4.55)$}} & \multirow{2}{*}{$<0.001$} \\
\hline & Control & $32.93 \pm 2.90$ & & & \\
\hline \multirow{2}{*}{ Hct. diff (Before-After) (\%) } & Case & $1.19 \pm 0.82$ & \multirow{2}{*}{\multicolumn{2}{|c|}{$-2.44(-3.00--1.88)$}} & \multirow{2}{*}{$<0.001$} \\
\hline & Control & $3.64 \pm 1.98$ & & & \\
\hline
\end{tabular}

*Mann-Whitney U. 
of hysteroscopy outcomes such as surgery duration, intraoperative bleeding, and distending media use will improve.

\section{Limitations of the Study}

In our study, bleeding categories were created based on the surgeon and nurse's estimation of the bleeding volume, which was clinically valuable. However, we did not evaluate the size, type, and accurate numbers of leiomyoma and endometrial polyps in the study; this information may affect the amount of bleeding. Future studies may provide more information by using quantitative estimates of bleeding and details about leiomyoma and endometrial polyps.

\section{Conclusions}

This study showed that removing clots and other uterine contents before insertion of the hysteroscope in patients with active uterine bleeding results in better and faster access to the uterine wall for observing the abnormalities and performing the appropriate procedure. This method is efficient and practical. There have been no similar studies regarding uterine evacuation during hysteroscopy in nonpregnant women with active uterine bleeding have been done. This method's success rate and effectiveness could be retested with different clinically important outcomes such as incomplete lesion resection and intrauterine adhesions.

\section{Authors' Contribution}

MS and MK designed the study and conducted the research. NB was the gynecology resident and aided in procedures. MS and MK monitored, evaluated, and analyzed the result of the study. Further, MS and MK took the lead in preparing the first draft of the report. Finally, MS, MK, PG, and VR reviewed the article. All authors approved the final manuscript and took responsibility for the integrity of the data..

\section{Conflict of Interests}

Authors declare that they have no conflict of interests.

\section{Ethical Issues}

The Ethics Committee of Tabriz University of Medical Sciences, Tabriz, Iran approved the study proposal (Code: IR.TBZMED.REC.1397.612). The study was fully described for all participants, and written informed consent was obtained from each woman with information confidentiality guarantees. Also the study protocol was registered in the Iranian Registry of Clinical Trials Center (identifier: IRCT20101130005283N13).

\section{Financial Support}

This study was funded by a Women's Reproductive Health Research Centre, Tabriz University of Medical Sciences, Tabriz, Iran.

\section{Acknowledgments}

The authors would like to thank all the participants for participating in this study.

\section{References}

1. Munro MG, Critchley HOD, Fraser IS. The two FIGO systems for normal and abnormal uterine bleeding symptoms and classification of causes of abnormal uterine bleeding in the reproductive years: 2018 revisions. Int J Gynaecol Obstet. 2018;143(3):393-408. doi:10.1002/ijgo.12666

2. Whitaker L, Critchley HO. Abnormal uterine bleeding. Best Pract Res Clin Obstet Gynaecol. 2016;34:54-65. doi:10.1016/j. bpobgyn.2015.11.012

3. Munro MG. Investigation of women with postmenopausal uterine bleeding: clinical practice recommendations. Perm J. 2014;18(1):55-70. doi:10.7812/tpp/13-072

4. Valentine LN, Bradley LD. Hysteroscopy for abnormal uterine bleeding and fibroids. Clin Obstet Gynecol. 2017;60(2):231-244. doi:10.1097/grf.0000000000000287

5. Sinha P, Yadav N, Gupta U. Use of hysteroscopy in abnormal uterine bleeding: an edge over histopathological examination. J Obstet Gynaecol India. 2018;68(1):45-50. doi:10.1007/s13224017-1065-5

6. Vilos GA, AlJasser R, Vilos AG, Oraif A, Abduljabar H, Abu-Rafea B. Histopathology and clinical outcomes of 151 women with postmenopausal bleeding treated with resectoscopic surgery. J Minim Invasive Gynecol. 2020;27(3):763-773. doi:10.1016/j. jmig.2019.06.004

7. Levy-Zauberman $\mathrm{Y}$, Pourcelot AG, Capmas $\mathrm{P}$, Fernandez $\mathrm{H}$. Update on the management of abnormal uterine bleeding. J Gynecol Obstet Hum Reprod. 2017;46(8):613-622. doi:10.1016/j. jogoh.2017.07.005

8. Cholkeri-Singh A, Sasaki KJ. Hysteroscopy safety. Curr Opin Obstet Gynecol. 2016;28(4):250-254. doi:10.1097/ gco.0000000000000289

9. Aas-Eng MK, Langebrekke A, Hudelist G. Complications in operative hysteroscopy - is prevention possible? Acta Obstet Gynecol Scand. 2017;96(12):1399-1403. doi:10.1111/ aogs.13209

10. Samy A, Raslan AN, Talaat B, et al. Perioperative nonhormonal pharmacological interventions for bleeding reduction during open and minimally invasive myomectomy: a systematic review and network meta-analysis. Fertil Steril. 2020;113(1):224-233.e6. doi:10.1016/j.fertnstert.2019.09.016

11. Hoffman CR, Van Hal M, Tariq R, George S. Complications of fluid overload during hysteroscopic surgery. Korean J Anesthesiol. 2019;72(4):387-388. doi:10.4097/kja.d.18.00374

12. Kamath MS, Kalampokas EE, Kalampokas TE. Use of GnRH analogues pre-operatively for hysteroscopic resection of submucous fibroids: a systematic review and meta-analysis. Eur J Obstet Gynecol Reprod Biol. 2014;177:11-18. doi:10.1016/j. ejogrb.2014.03.009

13. Lethaby A, Puscasiu L, Vollenhoven B. Preoperative medical therapy before surgery for uterine fibroids. Cochrane Database Syst Rev. 2017;11(11):CD000547. doi:10.1002/14651858. CD000547.pub2

14. Bizzarri N, Ghirardi V, Remorgida V, Venturini PL, Ferrero S. Threemonth treatment with triptorelin, letrozole and ulipristal acetate before hysteroscopic resection of uterine myomas: prospective comparative pilot study. Eur J Obstet Gynecol Reprod Biol. 2015;192:22-26. doi:10.1016/j.ejogrb.2015.06.018

15. Rasheedy R, Makled A, Abou-Gamrah A, Giuma H. Intrauterine instillation of tranexamic acid in hysteroscopic myomectomy: a double-blind, placebo-controlled, parallel-group randomized clinical trial. J Minim Invasive Gynecol. 2020;27(6):1264-1272. e2. doi:10.1016/j.jmig.2019.09.773

16. Shokeir T, El-Lakkany N, Sadek E, El-Shamy M, Abu Hashim H. An RCT: use of oxytocin drip during hysteroscopic endometrial resection and its effect on operative blood loss and glycine deficit. J Minim Invasive Gynecol. 2011;18(4):489-493. doi:10.1016/j. jmig.2011.03.015

17. Yu D, Li TC, Xia E, Huang X, Zheng J. A prospective randomized controlled trial on the effectiveness of routine Foley balloon tamponade on the reduction of bleeding after hysteroscopic resection of myoma. Gynecol Surg. 2006;3(2):93. doi:10.1007/ s10397-006-0188-y 
18. Cheng C, Zhao T, Xue M, Wan Y, Xu D. Use of suction curettage in operative hysteroscopy. J Minim Invasive Gynecol. 2009;16(6):739742. doi:10.1016/j.jmig.2009.07.010

19. Huchon C, Koskas M, Agostini A, et al. Operative hysteroscopy versus vacuum aspiration for incomplete spontaneous abortion (HY-PER): study protocol for a randomized controlled trial. Trials. 2015;16:363. doi:10.1186/s13063-015-0900-1

20. Raimondo G, Raimondo D, D'Aniello G, et al. A randomized controlled study comparing carbon dioxide versus normal saline as distension media in diagnostic office hysteroscopy: is the distension with carbon dioxide a problem? Fertil Steril. 2010;94(6):2319-2322. doi:10.1016/j.fertnstert.2009.11.041

21. Falcone F, Raimondo G, Stark M, Dessole S, Torella M, Raimondo I. Balloon catheter for cervical priming before operative hysteroscopy in young women: a pilot study. J Invest Surg. 2020;33(4):301-306. doi:10.1080/08941939.2018.1503379

22. Raimondo G, Grifone G, Raimondo D, Seracchioli R, Scambia G, Masciullo V. Hysteroscopic treatment of symptomatic cesareaninduced isthmocele: a prospective study. J Minim Invasive
Gynecol. 2015;22(2):297-301. doi:10.1016/j.jmig.2014.09.011

23. Indraccolo $U$, Bini $V$, Favilli A. Likelihood of accomplishing an in-patient hysteroscopic myomectomy in a one-step procedure: a systematic review and meta-analysis. Biomed Res Int 2020;2020:4208497. doi:10.1155/2020/4208497

24. Yendru KS, Yelamanchi SD, Vaddiraju GB. Hysteroscopic resection of submucous fibroids in symptomatic women. J Obstet Gynaecol India. 2019;69(2):166-172. doi:10.1007/s13224-018-1139-z

25. Lee $\mathrm{MMH}$. Endometrial polyp removed by a manual hysteroscopic tissue removal device. Gynecol Minim Invasive Ther. 2020;9(1):3435. doi:10.4103/gmit.gmit_116_18

26. Murakami T, Tamura M, Ozawa Y, Suzuki H, Terada Y, Okamura K. Safe techniques in surgery for hysteroscopic myomectomy. J Obstet Gynaecol Res. 2005;31(3):216-223. doi:10.1111/j.14470756.2005.00274.x

27. Zhu KA, Huang $H$, Xue $M$, et al. Removal of retained adherent placental remnants using the hysteroscopy endo-operative system. J Minim Invasive Gynecol. 2016;23(5):670-671. doi:10.1016/j. jmig.2016.01.027

(C) 2022 The Author(s); This is an open-access article distributed under the terms of the Creative Commons Attribution License (http:// creativecommons.org/licenses/by/4.0), which permits unrestricted use, distribution, and reproduction in any medium, provided the original work is properly cited. 